\title{
Magnetic Stability of Novel Exchange Coupled Systems
}

\author{
Materials Science Division \\ Argonne National Laboratory, Argonne, IL 60439
}

Submitted for the Proceedings of the 46th International Symposium of the American Vacuum Society, Seattle, WA, October 25-29, 1999

Work supported by the U.S. Department of Energy Basic Energy Sciences-Materials Sciences under contract \#W-31-109-ENG-38. 


\title{
Magnetic Stability of Novel Exchange Coupled Systems
}

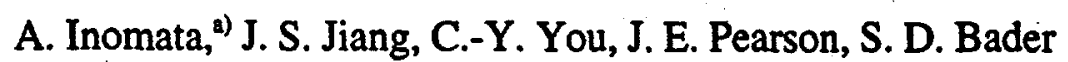 \\ Materials Science Division, Argonne National Laboratory, Argonne Illinois 60439
}

The magnetic stability of two different interfacial exchange coupled systems are investigated using the magneto-optic Kerr effect during repeated reversal of the soft layer magnetization by field cycling up to $10^{7}$ times. For $\mathrm{Fe} / \mathrm{Cr}$ 'double-superlattice' exchange biased systems, small but rapid initial decay of exchange bias field $\mathrm{H}_{\mathrm{E}}$ and the remanent magnetization is observed. Also the $\mathrm{Sm}-\mathrm{Co} / \mathrm{Fe}$ bilayers grown epitaxially with uniaxial in-plane anisotropy show similar decay. However, the $H_{E}$ of biaxial and random in-plane bilayers shows gradual decay without large reduction of the magnetization. These different decay behaviors explained by their different microstructure and interfacial spin configurations.

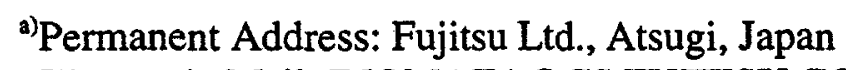

Electronic Mail: INOMATA@CS.FUITSU.CO.JP 


\section{DISCLAIMER}

This report was prepared as an account of work sponsored by an agency of the United States Government. Neither the United States Government nor any agency thereof, nor any of their employees, make any warranty, express or implied, or assumes any legal liability or responsibility for the accuracy, completeness, or usefulness of any information, apparatus, product, or process disclosed, or represents that its use would not infringe privately owned rights. Reference herein to any specific commercial product, process, or service by trade name, trademark, manufacturer, or otherwise does not necessarily constitute or imply its endorsement, recommendation, or favoring by the United States Government or any agency thereof. The views and opinions of authors expressed herein do not necessarily state or reflect those of the United States Government or any agency thereof. 


\section{DISCLAIMER}

Portions of this document may be illegible in electronic image products. Images are produced from the best available original document. 


\section{INTRODUCTION}

The application of interfacial exchange coupling is attractive to control magnetic properties ${ }^{1}$. Because of the ever-decreasing size of magnetic devices to the nanometer scale, a serious concern in these applications is the stability of the magnetization. Instabilities caused by thermal fluctuations are one of the biggest problems in the magnetic recording industry. ${ }^{2}$ Such instabilities in principle could be remedied by utilizing suitable exchange couplings. One example of this is that the exchange bias effect that occurs at the interface between a ferromagnet (F) and an antiferromagnet (AF) pinning layer is being used in new GMR (giant magnetoresistance) heads in high-density magnetic recording. ${ }^{3}$ Another example is that exchange-spring magnet nanostructures consisting of exchange coupled soft ferromagnets pinned to hard ferromagnetic phases are candidates for the next generation of high-energyproduct permanent magnet materials. ${ }^{4.5}$ Instabilities have also recently been reported in tunneling magnetoresistance (TMR) junctions of the hard reference layer against magnetization decay during field cycling of the adjacent soft layer. ${ }^{6}$

In this paper we present a comparison of the magnetic stability after multiple switching cycles in a novel exchange bias structure and exchange spring heterostructures. Our exchange bias system is an $\mathrm{Fe} / \mathrm{Cr}$ 'double superlattice' consisting of a ferromagnetic $\mathrm{Fe} / \mathrm{Cr}$ superlattice and an antiferromagnetic superlattice coupled through a $\mathrm{Cr}$ spacer. ${ }^{7} \mathrm{Fe} / \mathrm{Cr}$ double superlattices behave like conventional exchange bias systems, but with more coherent interfaces and controllable coupling strengths. For the exchange spring system we use $\mathrm{Sm}-\mathrm{Co} / \mathrm{Fe}$ bilayer structures grown epitaxially on different substrates to yield uniaxial, biaxial and random in-plane anisotropies. The epitaxially grown Sm-Co films have coercivity values of up to $4 \mathrm{~T}$ at room temperature. The 
switching fields and remanent magnetization of both systems were measured by the magnetooptic Kerr effect during repeated reversals of the soft layer magnetization by field cycling. The magnetic stability after $10^{7}$ cycles is reported and the effects of microstructure and interfacial spin configuration of the pinning layers are discussed.

\section{EXPERIMENT}

$\mathrm{Fe} / \mathrm{Cr}$ double superlattices and $\mathrm{Sm}-\mathrm{Co} / \mathrm{Fe}$ exchange spring bilayers were grown epitaxially by dc magnetron sputtering. In all cases, $\mathrm{Cr}$ buffer layers $200-\AA$ thick were deposited under suitable conditions to provide appropriate growth templates.

For the $\mathrm{Fe} / \mathrm{Cr}$ double superlattices, the $\mathrm{Cr}$ buffer layer was deposited onto single-crystal $\mathrm{MgO}(110)$ substrates at $400^{\circ} \mathrm{C}$ in an $\mathrm{Ar}$ pressure of $4 \mathrm{mTorr}$ to achieve (211)-oriented epitaxial growth. This template yields a strong in-plane uniaxial anisotropy in $\mathrm{Fe} / \mathrm{Cr}$ superlattices along $\mathrm{MgO}[0 \overline{1} 1]$ direction ${ }^{8}$. The substrate was then cooled to $100^{\circ} \mathrm{C}$ and the double-superlattices were grown in 2 mTorr of $\operatorname{Ar}$ as $[\mathrm{Fe}(14 \AA) / \operatorname{Cr}(11 \AA)]_{20} / \operatorname{Cr}(20 \AA) /[\mathrm{Fe}(50 \AA) / \operatorname{Cr}(20 \AA)]_{5}$ with a 50- $\AA \mathrm{Cr}$ capping layer. The $11-\AA \mathrm{Cr}$ and $20-\AA \mathrm{Cr}$ give rise to $\mathrm{AF}$ and $\mathrm{F}$ coupled $\mathrm{Fe} / \mathrm{Cr}$ superlattices, respectively. The $20-\AA \mathrm{Cr}$ between the two superlattices provides $\mathrm{F}$ coupling.

For the exchange spring bilayers, single-crystal $\mathrm{MgO}(100)$ and (110) substrates were used to grow (100) and (211)-oriented $\mathrm{Cr}$ epitaxial buffer layers, and glass substrates were used to grow (100)-textured $\mathrm{Cr}$ buffer layers at $150^{\circ} \mathrm{C}$ in $2.5 \mathrm{mTorr}$ of Ar. After depositing the $\mathrm{Cr}$ buffer layer, the substrates were kept at $400^{\circ} \mathrm{C}$ and the Sm-Co layers were epitaxially grown with subsequent deposition of an $800-\AA$ Fe layer. The Sm-Co/Fe bilayers were capped by $100 \AA$ of Cr. The Sm-Co films grown on $\operatorname{Cr}(211)$ and $\operatorname{Cr}(100)$ buffers on single crystal $\mathrm{MgO}$ substrates are (1 100$)$ - and $(11 \overline{2} 0)$-oriented with in-plane uniaxial and biaxial anisotropies, ${ }^{9}$ whereas the 
ones grown on (100)-textured $\mathrm{Cr}$ buffer layers on glass are (1120)-oriented but with randomly distributed easy axes in the film plane. The thickness of the Sm-Co layer was varied from 50 to $800 \AA$.

The exchange spring behavior of Sm-Co/Fe bilayers and the exchange bias characteristics of $\mathrm{Fe} / \mathrm{Cr}$ double-superlattices were confirmed via minor loop measurements using a Quantum Design dc magnetometer equipped with a 7-Tesla magnet and a magneto-optic Kerr effect (MOKE) setup with a 2-T magnet. With the MOKE system, the magnetic stability after multiple field cycles was measured from the change of switching fields and intensities of the minor loops. Due to the finite penetration depth of light $(\sim 200 \AA)$, the intensity of the MOKE signal is proportional to the magnetization of the F superlattice in the double superlattices, or to that of $\mathrm{Fe}$ layer in the exchange spring bilayers. The samples were initially magnetized to $+7 \mathrm{~T}$ along the easy axis direction and then the reverse field was cycled from 0 Oe to -260 Oe up to $10^{7}$ times at about $66 \mathrm{~Hz}$ using an air-gap coil. No magnetizing field was applied during the measurements and field cycles. To make sure that changes in measured signal are due to repeated switching of the free layer and not to a natural aging, the time dependence of $H_{E}$ and the magnetization were measured. The Sm-Co(50 $\mathrm{A}) / \mathrm{Fe}(800 \AA)$ film was kept in a field of -250 Oe for more than two days after being magnetized at $+7 \mathrm{~T}$. The remanent magnetization was measured periodically. To minimize the number of switching, only a few minor loops were measured. Even after $2 \times 10^{4}$ seconds, which is comparable to the time needed to measure the switching stability to $10^{7} \mathrm{cycles}$, no significant decay was found.

\section{RESULTS AND DISCUSSION}


Figure 1 shows the change in exchange bias with the number of field cycles $\mathrm{N}$ for an $\mathrm{Fe} / \mathrm{Cr}$ double-superlattice and uniaxial, biaxial and random in-plane $\mathrm{Sm}-\mathrm{Co}(800 \AA) / \mathrm{Fe}(800 \AA)$ bilayers. The exchange bias $\mathrm{H}_{E}$ is defined as the shift of the minor loop from zero field. $\mathrm{H}_{\mathrm{E} 0}$ is obtained from the first loop and $\Delta H_{E}=H_{E}-H_{E 0}$. The curves are normalized by $H_{E 0}$. From Figure 1, two kinds of decay characteristics can be seen. The two uniaxial systems-the $\mathrm{Fe} / \mathrm{Cr}$ double superlattice and the uniaxial bilayer-both show a rapid initial decay in $\mathrm{H}_{E}$ and then stabilize. The decay is strong $(\sim 3 \%-9 \%)$ for the first 10 cycles. On the other hand, the biaxial and the inplane random $\mathrm{Sm}-\mathrm{Co} / \mathrm{Fe}$ bilayers show a much slower decay in $\mathrm{H}_{\mathrm{E}}$, which does not stabilize even after $10^{7}$ field cycles. In the following, we will focus on the uniaxial and the biaxial $\mathrm{Sm}-\mathrm{Co} / \mathrm{Fe}$ bilayers for the two kinds of decay behaviors.

The observed decay patterns are similar to the 'training effect' in conventional AF/F exchange bias systems ${ }^{10}$ where the decrease in $\mathrm{H}_{E}$ is related to partial reorientation of the $A F$ domains with each FM magnetization reversal. ${ }^{1}$ The same mechanism could be at play here. The different decay behaviors in the uniaxial and the biaxial samples could be accounted for by noting the differences in microstructure and spin configurations at the interface of the pinning layers. High-resolution electron microscopy study ${ }^{11}$ shows that, due to local departure from the nominal stoichiometry during growth, the uniaxial $\mathrm{Sm}-\mathrm{Co}$ film has $\mathrm{SmCo}_{3}, \mathrm{Sm}_{2} \mathrm{Co}_{7}$ and $\mathrm{SmCo}_{5}$ polytypoids with stacking faults parallel to the hcp basal plane. These different phases have different magnetic anisotropy and share a common easy axis. Upon field cycling, regions with lower magnetic anisotropy become reversed, giving rise to the rapid initial decay in $\mathrm{H}_{\mathrm{E}}$. However, the planar stacking faults provide effective domain-wall pinning which prevents further propagation of reversed domains, and $\mathrm{H}_{\mathrm{E}}$ reaches saturation. The biaxial Sm-Co, on the other hand, has a bicrystalline microstructure with grains separated by incoherent boundaries. 
The grains have their easy axes in either $\mathrm{MgO}$ [001] or [010] directions. The strong intergranular coupling results in clusters of grains that have effective easy axes along either $\mathrm{MgO}$ [011] or $\mathrm{MgO}[0 \overline{1} 1]$ directions. It is likely that during repeated field cycling, the magnetization of some of the grains relax into their local preferred directions. Since this process involves only a $45^{\circ}$ rotation, the decrease in the coupling with the $\mathrm{Fe}$ layer would be small. This is evidenced by the smaller change of $\mathrm{H}_{\mathrm{E}}$ for the biaxial samples than for the uniaxial samples. The high density of twin boundaries could account for the very slow decay processes.

Figures 2 (a) and 2(b) show the decay behavior of the uniaxial and biaxial samples with different Sm-Co thicknesses. For the uniaxial samples, the one with $800 \AA \mathrm{Sm}$-Co shows strongest initial decay of $\mathrm{H}_{\mathrm{E}}$ and the rate of decay decreases with decreasing Sm-Co thickness. This behavior could be due to the island growth mode of Sm-Co. ${ }^{9}$ As the Sm-Co thickness increases, the roughness, and therefore inhomogeneity in the coupling with the Fe layer, increases. The initial decay in $\mathrm{H}_{\mathrm{E}}$ becomes stronger as a result. On the other hand, the decay of the nucleation field $H_{N}$ in the biaxial samples is less sensitive to the Sm-Co layer thickness. This is again consistent with the fact the density of twin boundaries in biaxial Sm-Co is thicknessindependent.

For the uniaxial samples with $800 \AA$ and $200 \AA \mathrm{Sm}-\mathrm{Co}$, the variation $\left(\mathrm{H}_{E}-\mathrm{H}_{E_{\infty}}\right)$ is proportional to $1 / \sqrt{\log N}$ for the first couple of hundred cycles, as Paccard et al. ${ }^{10}$ had shown. However, the bias field of the bilayer sample with $50 \AA \mathrm{Sm}$-Co decreases linearly with the number cycles. This is due to the gradual demagnetization of the thin Sm-Co layer. Shown in Figure 3 are the minor loops of the bilayer film after $1,100,10^{6}, 3 \times 10^{6}$, and $10^{7}$ field cycles. It is seen that only does the magnitude of the magnetization decrease, the squareness of the loop 
decreases as well. Since its thickness, $50 \AA$, is smaller than the thickness of a domain wall, the Sm-Co would be susceptible to irreversible switching by reversal of the coupled Fe layer. ${ }^{5}$

Figure 4 shows the minor loops of a biaxial Sm-Co(200 $\AA) / \mathrm{Fe}(800 \AA)$ bilayer sample after various numbers of field cycling. The demagnetizing branches gradually shift toward lower fields, but the recoil curves remain mostly unchanged. The shapes of the two branches are highly asymmetric. The asymmetry indicates different magnetization reversal mechanisms. According to Nikitenko et al. ${ }^{12}$ in the demagnetization process the magnetization is reversed sharply by a domain wall running through the Fe layer from edge to edge, while in the recoil process the magnetization returns to its original direction at nucleation centers throughout the film, located probably at grain boundaries. Since the propagation of a domain wall in the demagnetization branch involves the participation of all domains in the pinning layer, their gradual relaxation leads to the decrease of the switching field. However, since the locations of the nucleation centers are not likely to change, on average, the recoil curves would remain the same.

\section{SUMMARY}

The magnetic stability of two novel interfacial exchange coupled systems was investigated. For $\mathrm{Fe} / \mathrm{Cr}$ double-superlattice and exchange spring bilayers with uniaxial $\mathrm{Sm}-\mathrm{Co}$, the exchange bias field $\mathrm{H}_{\mathrm{E}}$ shows strong initial decay and then becoming stable as in a training effect. However, for the biaxial samples, $H_{E}$ decays gradually and does not reach saturation. The different decay behaviors are attributed to the different microstructure and spin configuration of the pinning layers. The strong initial decay in uniaxial samples are due to the reversal local inhomogeneities whereas the gradual decay in biaxial samples are due to slow relaxation metastable spin configuration. For applications that require long-term stability in the exchange 
bias field, the uniaxial pinning layers might be preferred. The asymmetric hysteresis loops of the biaxial samples indicate different mechanisms for the demagnetization and recoil processes. These results help us to begin to understand the magnetic stability in terms of the stability of the crystallographic microstructure. However, such studies in the future would benefit enormously from direct microstructural observations to verify these ideas.

\section{ACKNOWLEDGMENTS}

Work at Argonne is supported by the U.S. Department of Energy, Basic Energy SciencesMaterials Sciences, under Contract No. W-31-109-ENG-38. We thank E. E. Fullerton for providing glass substrates and A. Berger for valuable suggestions. We also thank Fujitsu Ltd. for its kind support. 


\section{FIGURE CAPTIONS}

Fig. 1 The change in exchange bias $\Delta \mathrm{H}_{E}$ with numbers of field cycles $\mathrm{N}$ for an $[\mathrm{Fe}(14 \AA) / \mathrm{Cr}(11$ $\AA)]_{20} / \operatorname{Cr}(20 \AA) /[\mathrm{Fe}(50 \AA) / \operatorname{Cr}(20 \AA)]_{5}$ double superlattice and $\mathrm{Sm}-\mathrm{Co}(800 \AA) / \mathrm{Fe}(800 \AA)$ exchange spring bilayers with uniaxial, biaxial and random in-plane SmCo layer. The curves are normalized by the initial exchange bias field value denoted $\mathrm{H}_{\mathrm{E} 0}$.

Fig. 2 (a) The change in exchange bias $\Delta \mathrm{H}_{\mathrm{E}}$ with $\mathrm{N}$ for uniaxial $\mathrm{Sm}-\mathrm{Co} / \mathrm{Fe}(800 \AA)$ bilayers with different Sm-Co thicknesses. (b) The change in nucleation field $\mathrm{H}_{\mathrm{N}}$ with $\mathrm{N}$ for biaxial Sm-Co/Fe $(800 \AA)$ bilayers with different Sm-Co thicknesses. $H_{N}$ is defined as the switching field in the demagnetization curve.

Fig. 3 The minor hysteresis loops of the uniaxial Sm-Co(50 $\AA) / \mathrm{Fe}(800 \AA)$ bilayer after different number of field cycles.

Fig. 4 The demagnetization and recoil curves for the biaxial Sm-Co $(200 \AA) / \mathrm{Fe}(800 \AA)$ bilayer for different numbers of field cycles. 


\section{REFERENCES}

${ }^{1}$ J. Nogues, and I.K. Schuller, J. Magn. Magn. Mater. 192, 203 (1999).

${ }^{2}$ S.H. Charap, P.-L. Lu and Y. He, IEEE Trans. Magn. 33, 1, 978 (1997).

${ }^{3}$ B. Dieny, V.S. Speriosu, S.S.P. Parkin, B.A. Gurney, D.R. Wilhoit and D. Mauri, Phys. Rev. B. 43, 1, $1297(1991)$.

${ }^{4}$ E. F. Kneller and R. Hawig, IEEE Trans. Mag. 27, 3588 (1991).

${ }^{5}$ E. E. Fullerton, J. S. Jiang, M. Grimsditch, C. H. Sowers, and S. D. Bader, Phys. Rev. B 58, 12193 (1998).

${ }^{6}$ S. Gider, B.-U. Runge, A.C. Marley, and S.S.P. Parkin, Science 281, 797 (1998).

' J.S. Jiang et al. submitted.

${ }^{8}$ E.E. Fullerton, M.J. Conover, J.E. Mattson, C.H. Sowers and S.D. Bader, Phys. Rev. B. 48, 21, 15755 (1993).

${ }^{9}$ E. E. Fullerton, J. S. Jiang, C. Rehm, C. H. Sowers, S. D. Bader, J. B. Patel and X. Z. Wu, Appl. Phys. Lett. 71, 1579 (1997).

${ }^{10}$ D. Paccard, C. Schlenker, O, Massenet, R. Montmory, and A. Yelon, Phys. Stat. Sol. 16, 301 (1966).

"M. Benaissa, K.M. Krishnan, E.E. Fullerton, and J.S. Jiang, IEEE Trans. Magn. 34, 4, 1204 (1998). 
${ }^{12}$ V.I. Nikitenko, V.S. Gornakov, L.M. Dedukh, Y.P. Kabanov, A.F. Khapikov, A.J. Shapiro, R.D. Shull, A.Chaiken, and R.P. Michel, Phys. Rev. B. 57, 14, R8111 (1998). 


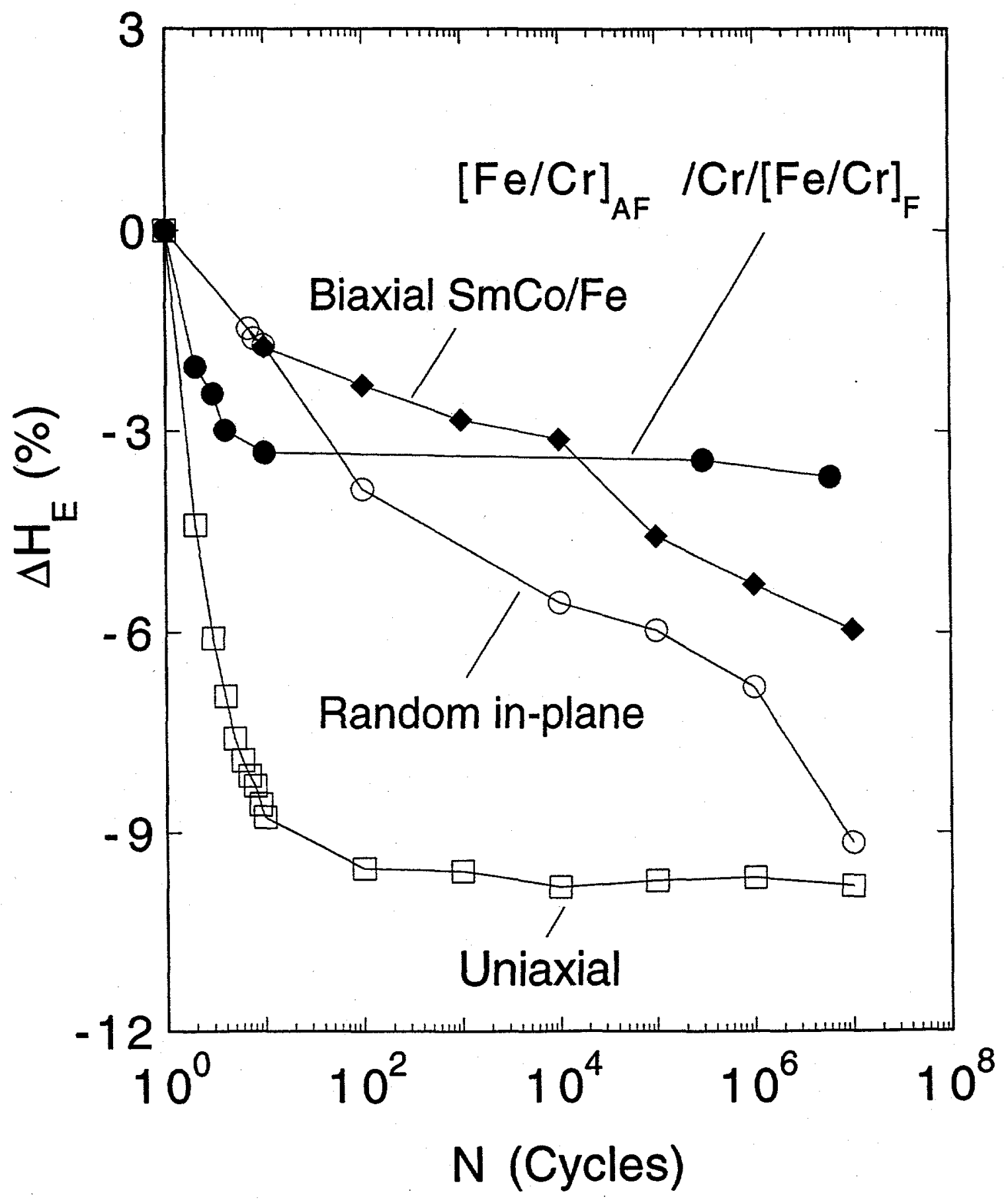

Fig. 1 
$\sum_{\substack{1 \\ 0}}^{+}$
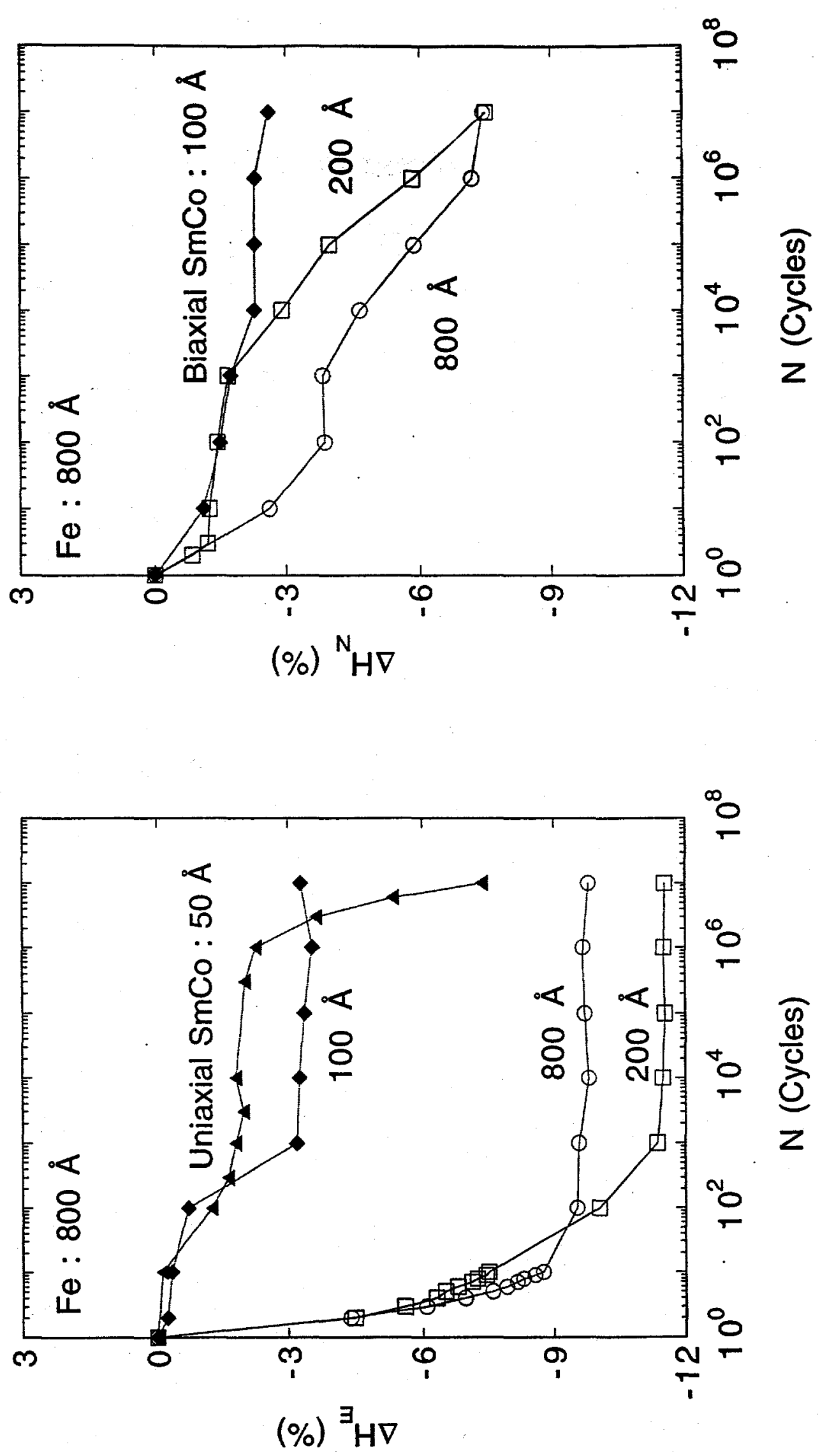


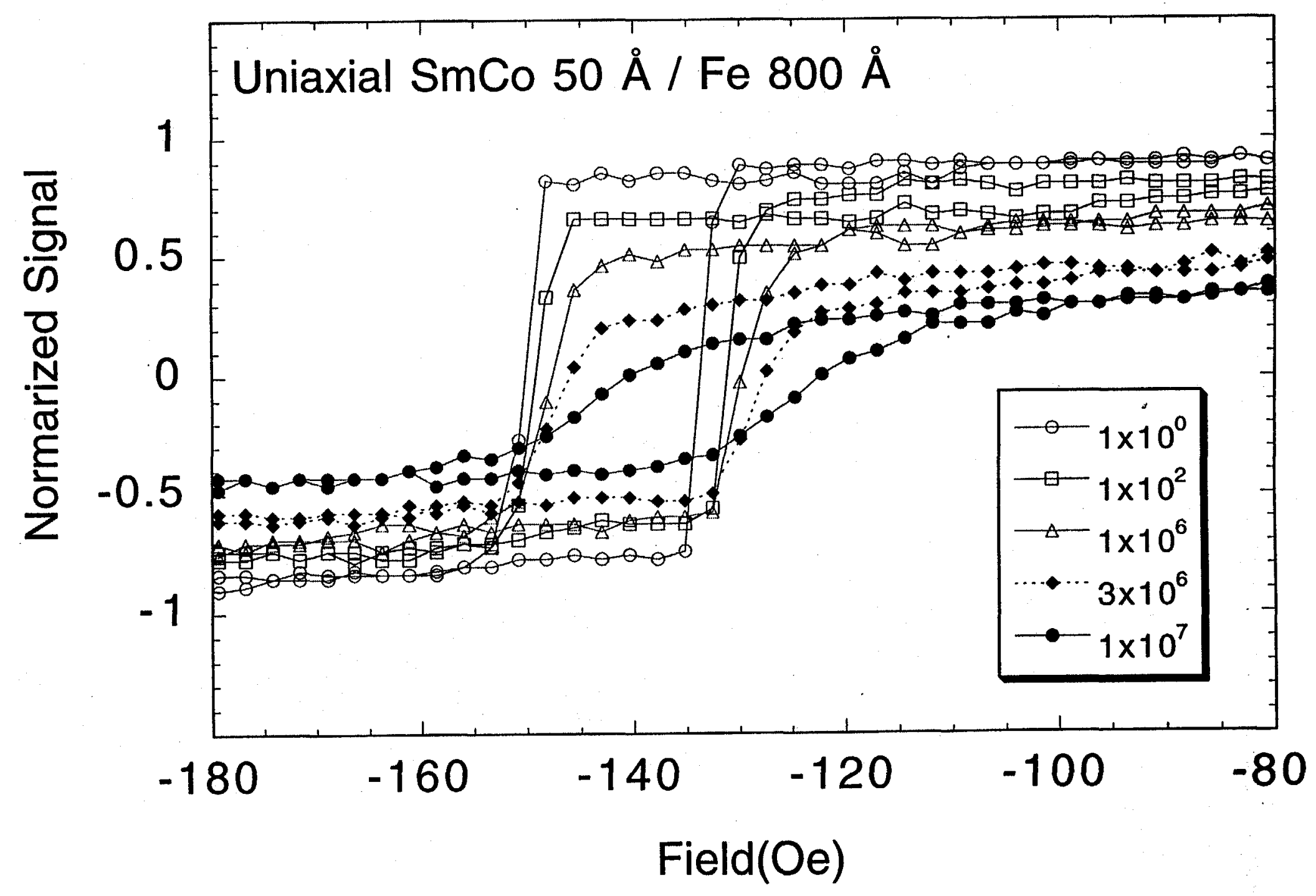

Fig. 3 


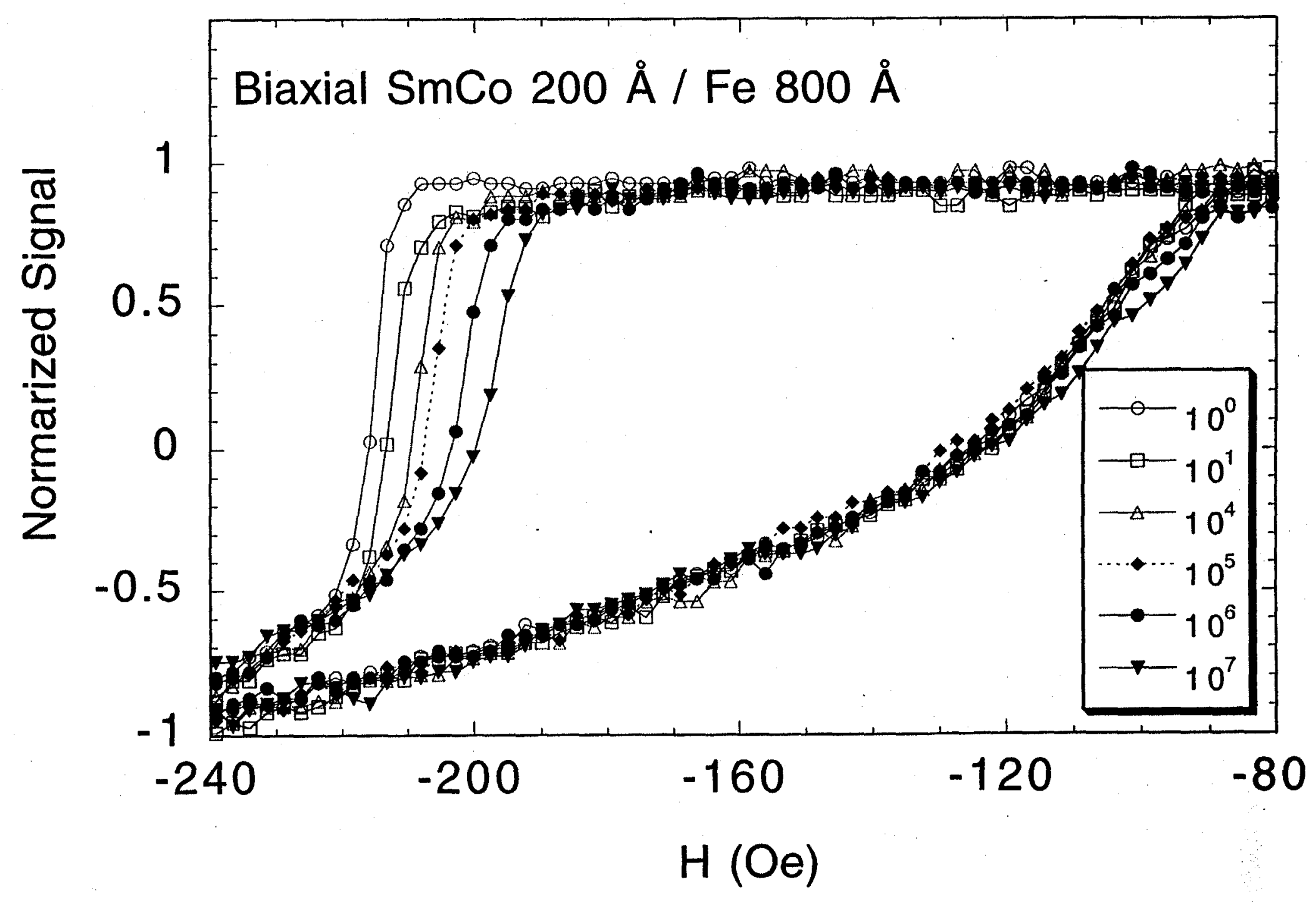

Fig. 4 